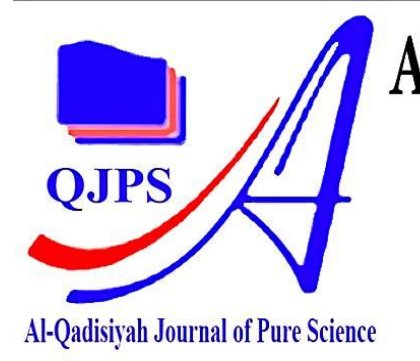

Al-Qadisiyah Journal of Pure Science

ISSN(Printed): 1997-2490

ISSN(Online):

$2411-3514$

DOI : /10.29350/jops.

http:// qu.edu.iq/journalsc/index.php/JOPS

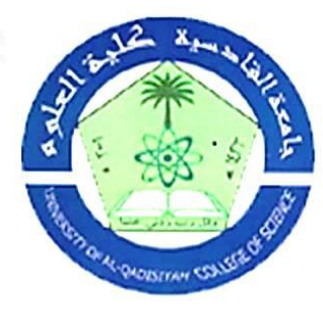

\title{
On Semigroup Ideals and Generalized Two Sided Reverse $\alpha$-3- Derivation in Prime Near-Ring
}

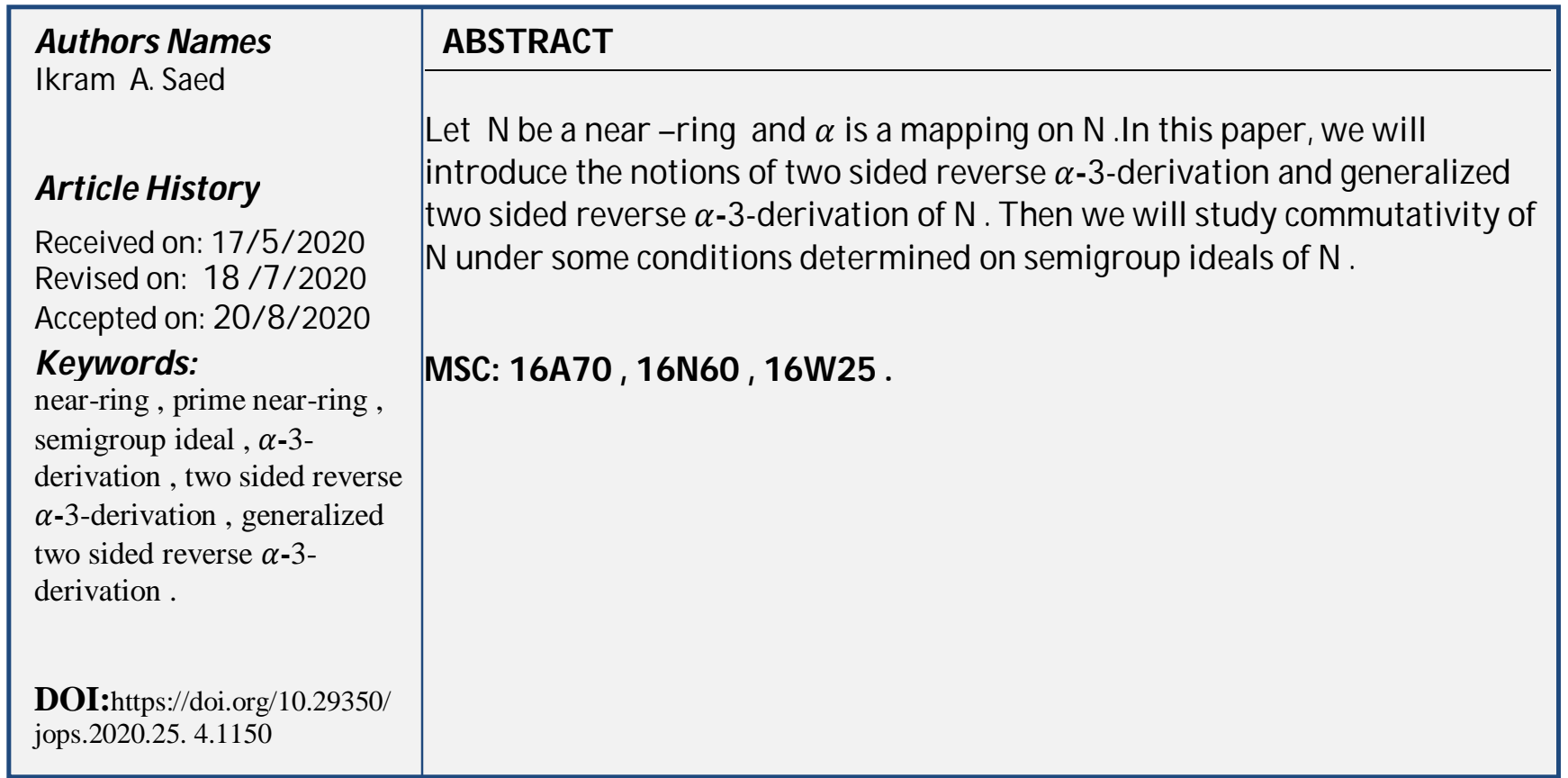

\section{Introduction}

N. Argac [6] studied the commutativity of prime near-ring $\mathrm{N}$ using the notion of two sided $\alpha$ derivation. N. L. Oukhtite and A. Raji [7] continued in the same line, they generalized some known results involving semigroup ideal and generalized two sided $\alpha$-derivations . M. Ashraf et al. $[8,9,10,11]$ studied the commutativity of near-ring $\mathrm{N}$ using the notions of $\mathrm{n}$-derivations , $(\sigma, \tau)$-n-derivations and generalized n-derivations. Hence, it should be interesting to study the commutativity of a near-ring $\mathrm{N}$ admitting some conditions on other $n$-additive mappings . E. F. Adhab [5] studied the commutativity of prime near-ring N using the notions of two sided $\alpha$-nderivation and generalized two sided $\alpha$-n-derivation of near-ring $\mathrm{N}$.

For more information see $[12,13,14]$

Let $\mathrm{N}$ be a near -ring and $\alpha$ is a mapping on $\mathrm{N}$. This paper consists of two section s. In section one, we recall some known definitions and necessary lemmas that we will use it later in this 
paper. In section two, we define the concepts of two sided reverse $\alpha$-3-derivation and generalized two sided reverse $\alpha$-3-derivation of $\mathrm{N}$, also we determine some conditions of generalized two sided reverse $\alpha$-3-derivation and semigroup ideals which make prime near-ring commutative ring .

\section{Basic Concepts}

Definition 2.1:[1] Aright near-ring (resp. a left near-ring ) is a nonempty set N equipped with two binary operations + and . such that

(i) $(\mathrm{N},+)$ is a group ( not necessarily abelian )

(ii) $(\mathrm{N}$, .) is a semigroup .

(iii) For all $x, y, z \in N$, we have

$$
(x+y) z=x z+y z(\operatorname{resp} \cdot z(x+y)=z x+z y)
$$

Example 2.2:[1] Let Gbe a group ( not necessarily abelian ) then all mapping of G into itself form a right near-ring $\mathrm{M}(\mathrm{G})$ with regard to point wise addition and multiplication by composite

Definition 2.3:[2] Anear-ring $\mathrm{N}$ is called a prime near-ring if $\mathrm{aNb}=\{0\}$, where $\mathrm{a}, \mathrm{b} \in \mathrm{N}$, implies that either $a=0$ or $b=0$.

Definition 2.4:[2] Let $\mathrm{N}$ be a near-ring . The symbol Zwill denote the multiplicative center of $\mathrm{N}$, that is $Z=\{x \in N / x y=y x$ for all $y \in N\}$.

Definition 2.5:[2] Let $\mathrm{R}$ be a ring. Define a Lie product [ , ] on $\mathrm{R}$ as follows

$[x, y]=x y-y x$, for all $x, y \in R$.

Properties 2.6:[2] Let $\mathrm{R}$ be a ring, then for all $\mathrm{x}, \mathrm{y}, \mathrm{z} \in \mathrm{R}$, we have :

$$
\begin{aligned}
& 1-[x, y z]=y[x, z]+[x, y] z \\
& 2-[x y, z]=x[y, z]+[x, z] y \\
& 3-[x+y, z]=[x, z]+[y, z] \\
& 4-[x, y+z]=[x, y]+[x, z]
\end{aligned}
$$

Definition 2.7:[2] A nonempty subset $\mathrm{U}$ of $\mathrm{N}$ will be called a semigroup right ideal ( resp. semigroup left ideal ) if $U N \subset U(\operatorname{resp} . N U \subset U$ ) and if $U$ is both semigroup right ideal and semigroup left ideal, it be called a semigroup ideal .

Remark $2.8:[2]$ Let $\mathrm{N}$ be a near-ring

(i) $\mathrm{N} \times \mathrm{Nx} \ldots \mathrm{x}$ forms a near-ring with regard to component wise addition and component wise multiplication . 
(ii) If $\mathrm{U}_{1}, \mathrm{U}_{2}, \ldots, \mathrm{U}_{\mathrm{n}}$ be nonzero semigroup right ideals ( resp. semigroup left ideals ) of $\mathrm{N}$, then $\mathrm{U}_{1} \times \mathrm{U}_{2} \mathrm{x} \ldots \mathrm{x} \mathrm{U}_{\mathrm{n}}$ forms a nonzero semigroup right ideals ( resp. semigroup left ideas ) of $\mathrm{N} \times \mathrm{N}$ x .... N .

Definition 2.9:[3] Suppose that $\mathrm{W}$ is a near-ring. An 3-additive mapping $\mathrm{d}: \mathrm{W} \times \mathrm{W} x \mathrm{~W} \rightarrow \mathrm{W}$ is called 3-derivation if the relations :

$\mathrm{d}\left(\mathrm{s}_{1} \mathrm{~s}_{1}^{\prime}, \mathrm{s}_{2}, \mathrm{~s}_{3}\right)=\mathrm{d}\left(\mathrm{s}_{1}, \mathrm{~s}_{2}, \mathrm{~s}_{3}\right) \mathrm{s}_{1}^{\prime}+\mathrm{s}_{1} \mathrm{~d}\left(\mathrm{~s}_{1}^{\prime}, \mathrm{s}_{2}, \mathrm{~s}_{3}\right)$

$\mathrm{d}\left(\mathrm{s}_{1}, \mathrm{~s}_{2} \mathrm{~s}_{2} /, \mathrm{s}_{3}\right)=\mathrm{d}\left(\mathrm{s}_{1}, \mathrm{~s}_{2}, \mathrm{~s}_{3}\right) \mathrm{s}_{2}^{\prime}+\mathrm{s}_{2} \mathrm{~d}\left(\mathrm{~s}_{1}, \mathrm{~s}_{2}^{\prime}, \mathrm{s}_{3}\right)$

$\mathrm{d}\left(\mathrm{s}_{1}, \mathrm{~s}_{2}, \mathrm{~s}_{3} \mathrm{~s}_{3}^{\prime}\right)=\mathrm{d}\left(\mathrm{s}_{1}, \mathrm{~s}_{2}, \mathrm{~s}_{3}\right) \mathrm{s}_{3}{ }^{\prime}+\mathrm{s}_{3} \mathrm{~d}\left(\mathrm{~s}_{1}, \mathrm{~s}_{2}, \mathrm{~s}_{3}^{\prime}\right)$

hold for all $\mathrm{s}_{1}, \mathrm{~s}_{1}^{\prime}, \mathrm{s}_{2}, \mathrm{~s}_{2}^{\prime}, \mathrm{s}_{3}, \mathrm{~s}_{3}^{\prime} \in \mathrm{W}$.

Example $2.10:[4]$ Let $\mathrm{S}$ be a commutative near-ring .

Let us define

$\mathrm{W}=\left\{\left(\begin{array}{ll}r & u \\ 0 & 0\end{array}\right): r, u, 0 \in S\right\}$.

And $\mathrm{d}: \mathrm{WxWx} \mathrm{W} \rightarrow \mathrm{W}$

$\mathrm{d}\left(\left(\begin{array}{cc}r_{1} & u_{1} \\ 0 & 0\end{array}\right),\left(\begin{array}{cc}r_{2} & u_{2} \\ 0 & 0\end{array}\right),\left(\begin{array}{cc}r_{3} & u_{3} \\ 0 & 0\end{array}\right)\right)=\left(\begin{array}{cc}0 & r_{1} r_{2} r_{3} \\ 0 & 0\end{array}\right)$

Then $\mathrm{d}$ is 3-derivation of $\mathrm{W}$.

Definition 2.11:[4] Suppose that $\mathrm{W}$ is a near-ring and d be 3-derivation of W. An 3-additive mapping $\mathrm{f}: \mathrm{WxWxW} \rightarrow \mathrm{W}$ is said to be generalized 3-derivation of $\mathrm{W}$ associated with $\mathrm{d}$ if the relations

$\mathrm{f}\left(\mathrm{s}_{1} \mathrm{~s}_{1}^{\prime}, \mathrm{s}_{2}, \mathrm{~s}_{3}\right)=\mathrm{f}\left(\mathrm{s}_{1}, \mathrm{~s}_{2}, \mathrm{~s}_{3}\right) \mathrm{s}_{1}^{\prime}+\mathrm{s}_{1} \mathrm{~d}\left(\mathrm{~s}_{1}^{\prime}, \mathrm{s}_{2}, \mathrm{~s}_{3}\right)$

$\mathrm{f}\left(\mathrm{s}_{1}, \mathrm{~s}_{2} \mathrm{~s}_{2}{ }^{\prime}, \mathrm{s}_{3}\right)=\mathrm{f}\left(\mathrm{s}_{1}, \mathrm{~s}_{2}, \mathrm{~s}_{3}\right) \mathrm{s}_{2}{ }^{\prime}+\mathrm{s}_{2} \mathrm{~d}\left(\mathrm{~s}_{1}, \mathrm{~s}_{2}{ }^{\prime}, \mathrm{s}_{3}\right)$

$\mathrm{f}\left(\mathrm{s}_{1}, \mathrm{~s}_{2}, \mathrm{~s}_{3} \mathrm{~s}_{3}{ }^{\prime}\right)=\mathrm{f}\left(\mathrm{s}_{1}, \mathrm{~s}_{2}, \mathrm{~s}_{3}\right) \mathrm{s}_{3}{ }^{\prime}+\mathrm{s}_{3} \mathrm{~d}\left(\mathrm{~s}_{1}, \mathrm{~s}_{2}, \mathrm{~s}_{3}{ }^{\prime}\right)$

hold for all $\mathrm{s}_{1}, \mathrm{~s}_{1}{ }^{\prime}, \mathrm{s}_{2}, \mathrm{~s}_{2}{ }^{\prime}, \mathrm{s}_{3}, \mathrm{~s}_{3}{ }^{\prime} \in \mathrm{W}$.

Example 2.12 :[4] Let $\mathrm{S}$ be a commutative near-ring .

Let us define

$\mathrm{W}=\left\{\left(\begin{array}{cc}0 & r \\ 0 & u\end{array}\right): r, u, 0 \in S\right\}$.

And $\mathrm{d}, \mathrm{f}: \mathrm{WxWxW} \rightarrow \mathrm{W}$,

$\mathrm{d}\left(\left(\begin{array}{ll}0 & r_{1} \\ 0 & u_{1}\end{array}\right),\left(\begin{array}{ll}0 & r_{2} \\ 0 & u_{2}\end{array}\right),\left(\begin{array}{ll}0 & r_{3} \\ 0 & u_{3}\end{array}\right)\right)=\left(\begin{array}{cc}0 & r_{1} r_{2} r_{3} \\ 0 & 0\end{array}\right)$

$\mathrm{f}\left(\left(\begin{array}{cc}0 & r_{1} \\ 0 & u_{1}\end{array}\right),\left(\begin{array}{ll}0 & r_{2} \\ 0 & u_{2}\end{array}\right),\left(\begin{array}{cc}0 & r_{3} \\ 0 & u_{3}\end{array}\right)\right)=\left(\begin{array}{cc}0 & 0 \\ 0 & u_{1} \\ u_{2} u_{3}\end{array}\right)$

Then $\mathrm{f}$ is a generalized 3 -derivation of $\mathrm{W}$.

Lemma $2.13:[5]$ Let $\mathrm{N}$ be a prime near-ring and $\mathrm{U}$ be a nonzero semigroup right ideal ( resp. semigroup left ideal ) of $\mathrm{N}$ and $\mathrm{x}$ is an element of $\mathrm{N}$ such that $U \mathrm{x}=\{0\}$ (resp. $\mathrm{xU}=\{0\})$, then $\mathrm{x}=0$.

Lemma 2.14:[5] Let $\mathrm{N}$ be a prime near-ring and $U$ be a nonzero semigroup ideal of $\mathrm{N}$. If $\mathrm{x}, \mathrm{y} \in \mathrm{N}$ and $\mathrm{xUy}=\{0\}$, then $\mathrm{x}=0$ or $\mathrm{y}=0$.

Lemma $2.15:[5]$ Let $\mathrm{N}$ be a prime near-ring and $\mathrm{Z}$ contains a nonzero semigroup left ideal or nonzero semigroup right ideal, then $\mathrm{N}$ is a commutative ring .

3.Main Results 
First we introduce the basic definitions in this paper

Definition 3.1: Let $\mathrm{N}$ be a near-ring .An 3-additive mapping $\mathrm{d}: \mathrm{N} \times \mathrm{Nx} \mathrm{N} \rightarrow \mathrm{N}$ is said to be two sided reverse $\alpha-3$-derivation if there exists a function $\alpha: \mathrm{N} \rightarrow \mathrm{N}$ such that the relations :

$$
\begin{array}{r}
\mathrm{d}\left(\mathrm{x}_{1}^{\prime} \mathrm{x}_{1}, \mathrm{x}_{2}, \mathrm{x}_{3}\right)=\mathrm{d}\left(\mathrm{x}_{1}, \mathrm{x}_{2}, \mathrm{x}_{3}\right) \mathrm{x}_{1}^{\prime}+\alpha\left(\mathrm{x}_{1}\right) \mathrm{d}\left(\mathrm{x}_{1}^{\prime}, \mathrm{x}_{2}, \mathrm{x}_{3}\right) \\
=\mathrm{d}\left(\mathrm{x}_{1}, \mathrm{x}_{2}, \mathrm{x}_{3}\right) \alpha\left(\mathrm{x}_{1}^{\prime}\right)+\mathrm{x}_{1} \mathrm{~d}\left(\mathrm{x}_{1}^{\prime}, \mathrm{x}_{2}, \mathrm{x}_{3}\right) \\
\mathrm{d}\left(\mathrm{x}_{1}, \mathrm{x}_{2} \mathrm{x}_{2}, \mathrm{x}_{3}\right)=\mathrm{d}\left(\mathrm{x}_{1}, \mathrm{x}_{2}, \mathrm{x}_{3}\right) \mathrm{x}_{2}^{\prime}+\alpha\left(\mathrm{x}_{2}\right) \mathrm{d}\left(\mathrm{x}_{1}, \mathrm{x}_{2}^{\prime}, \mathrm{x}_{3}\right) \\
=\mathrm{d}\left(\mathrm{x}_{1}, \mathrm{x}_{2}, \mathrm{x}_{3}\right) \alpha\left(\mathrm{x}_{2}^{\prime}\right)+\mathrm{x}_{2} \mathrm{~d}\left(\mathrm{x}_{1}, \mathrm{x}_{2}^{\prime}, \mathrm{x}_{3}\right) \\
\mathrm{d}\left(\mathrm{x}_{1}, \mathrm{x}_{2}, \mathrm{x}_{3}^{\prime} \mathrm{x}_{3}\right)=\mathrm{d}\left(\mathrm{x}_{1}, \mathrm{x}_{2}, \mathrm{x}_{3}\right) \mathrm{x}_{3}^{\prime}+\alpha\left(\mathrm{x}_{3}\right) \mathrm{d}\left(\mathrm{x}_{1}, \mathrm{x}_{2}, \mathrm{x}_{3}^{\prime}\right) \\
=\mathrm{d}\left(\mathrm{x}_{1}, \mathrm{x}_{2}, \mathrm{x}_{3}\right) \alpha\left(\mathrm{x}_{3}^{\prime}\right)+\mathrm{x}_{3} \mathrm{~d}\left(\mathrm{x}_{1}, \mathrm{x}_{2}, \mathrm{x}_{3}^{\prime}\right)
\end{array}
$$

hold for all $\mathrm{x}_{1}, \mathrm{x}_{1}^{\prime}, \mathrm{x}_{2}, \mathrm{x}_{2}^{\prime}, \mathrm{x}_{3}, \mathrm{x}_{3}^{\prime} \in \mathrm{N}$.

Definition 3.2: Let $\mathrm{N}$ be a near-ring and $\mathrm{d}: \mathrm{Nx} \mathrm{N} x \mathrm{~N} \rightarrow \mathrm{N}$ be a two sided reverse $\alpha$-3-derivation of $\mathrm{N}$. An 3-additive mapping $\mathrm{f}: \mathrm{N} \times \mathrm{N} \times \mathrm{N} \rightarrow \mathrm{N}$ is said to be generalized two sided reverse $\alpha$-3derivation associated with two sided reverse $\alpha$-3-derivation $\mathrm{d}$ if the relations :

$$
\begin{array}{r}
\mathrm{f}\left(\mathrm{x}_{1}^{\prime} \mathrm{x}_{1}, \mathrm{x}_{2}, \mathrm{x}_{3}\right)=\mathrm{d}\left(\mathrm{x}_{1}, \mathrm{x}_{2}, \mathrm{x}_{3}\right) \mathrm{x}_{1}^{\prime}+\alpha\left(\mathrm{x}_{1}\right) \mathrm{f}\left(\mathrm{x}_{1}^{\prime}, \mathrm{x}_{2}, \mathrm{x}_{3}\right) \\
=\mathrm{d}\left(\mathrm{x}_{1}, \mathrm{x}_{2}, \mathrm{x}_{3}\right) \alpha\left(\mathrm{x}_{1}^{\prime}\right)+\mathrm{x}_{1} \mathrm{f}\left(\mathrm{x}_{1}^{\prime}, \mathrm{x}_{2}, \mathrm{x}_{3}\right) \\
\mathrm{f}\left(\mathrm{x}_{1}, \mathrm{x}_{2}^{\prime} \mathrm{x}_{2}, \mathrm{x}_{3}\right)=\mathrm{d}\left(\mathrm{x}_{1}, \mathrm{x}_{2}, \mathrm{x}_{3}\right) \mathrm{x}_{2}^{\prime}+\alpha\left(\mathrm{x}_{2}\right) \mathrm{f}\left(\mathrm{x}_{1}, \mathrm{x}_{2}^{\prime}, \mathrm{x}_{3}\right) \\
=\mathrm{d}\left(\mathrm{x}_{1}, \mathrm{x}_{2}, \mathrm{x}_{3}\right) \alpha\left(\mathrm{x}_{2}^{\prime}\right)+\mathrm{x}_{2} \mathrm{f}\left(\mathrm{x}_{1}, \mathrm{x}_{2}^{\prime}, \mathrm{x}_{3}\right) \\
\mathrm{f}\left(\mathrm{x}_{1}, \mathrm{x}_{2}, \mathrm{x}_{3}^{\prime} \mathrm{x}_{3}\right)=\mathrm{d}\left(\mathrm{x}_{1}, \mathrm{x}_{2}, \mathrm{x}_{3}\right) \mathrm{x}_{3}^{\prime}+\alpha\left(\mathrm{x}_{3}\right) \mathrm{f}\left(\mathrm{x}_{1}, \mathrm{x}_{2}, \mathrm{x}_{3}^{\prime}\right) \\
=\mathrm{d}\left(\mathrm{x}_{1}, \mathrm{x}_{2}, \mathrm{x}_{3}\right) \alpha\left(\mathrm{x}_{3}^{\prime}\right)+\mathrm{x}_{3} \mathrm{f}\left(\mathrm{x}_{1}, \mathrm{x}_{2}, \mathrm{x}_{3}^{\prime}\right)
\end{array}
$$

hold for all $\mathrm{x}_{1}, \mathrm{x}_{1}^{\prime}, \mathrm{x}_{2}, \mathrm{x}_{2}^{\prime}, \mathrm{x}_{3}, \mathrm{x}_{3}^{\prime} \in \mathrm{N}$.

We begin with the following lemmas which are essential for developing the proofs of our main results,$\alpha$ will represent a homomorphism of $\mathrm{N}$.

Lemma 3.3 : Let $\mathrm{N}$ be a near-ring and $\mathrm{d}$ be a two sided reverse $\alpha$-3-derivation of $\mathrm{N}$, then

$$
\begin{array}{r}
\mathrm{d}\left(\mathrm{x}_{1}^{\prime} \mathrm{x}_{1}, \mathrm{x}_{2}, \mathrm{x}_{3}\right)=\mathrm{x}_{1} \mathrm{~d}\left(\mathrm{x}_{1}^{\prime}, \mathrm{x}_{2}, \mathrm{x}_{3}\right)+\mathrm{d}\left(\mathrm{x}_{1}, \mathrm{x}_{2}, \mathrm{x}_{3}\right) \alpha\left(\mathrm{x}_{1}^{\prime}\right) \\
=\alpha\left(\mathrm{x}_{1}\right) \mathrm{d}\left(\mathrm{x}_{1}^{\prime}, \mathrm{x}_{2}, \mathrm{x}_{3}\right)+\mathrm{d}\left(\mathrm{x}_{1}, \mathrm{x}_{2}, \mathrm{x}_{3}\right) \mathrm{x}_{1}^{\prime} \\
\mathrm{d}\left(\mathrm{x}_{1}, \mathrm{x}_{2} \mathrm{x}_{2}, \mathrm{x}_{3}\right)=\mathrm{x}_{2} \mathrm{~d}\left(\mathrm{x}_{1}, \mathrm{x}_{2}^{\prime}, \mathrm{x}_{3}\right)+\mathrm{d}\left(\mathrm{x}_{1}, \mathrm{x}_{2}, \mathrm{x}_{3}\right) \alpha\left(\mathrm{x}_{2}^{\prime}\right) \\
=\alpha\left(\mathrm{x}_{2}\right) \mathrm{d}\left(\mathrm{x}_{1}, \mathrm{x}_{2}^{\prime}, \mathrm{x}_{3}\right)+\mathrm{d}\left(\mathrm{x}_{1}, \mathrm{x}_{2}, \mathrm{x}_{3}\right) \mathrm{x}_{2}^{\prime} \\
\mathrm{d}\left(\mathrm{x}_{1}, \mathrm{x}_{2}, \mathrm{x}_{3}^{\prime} \mathrm{x}_{3}\right)=\mathrm{x}_{3} \mathrm{~d}\left(\mathrm{x}_{1}, \mathrm{x}_{2}, \mathrm{x}_{3}^{\prime}\right)+\mathrm{d}\left(\mathrm{x}_{1}, \mathrm{x}_{2}, \mathrm{x}_{3}\right) \alpha\left(\mathrm{x}_{3}^{\prime}\right) \\
=\alpha\left(\mathrm{x}_{3}\right) \mathrm{d}\left(\mathrm{x}_{1}, \mathrm{x}_{2}, \mathrm{x}_{3}^{\prime}\right)+\mathrm{d}\left(\mathrm{x}_{1}, \mathrm{x}_{2}, \mathrm{x}_{3}\right) \mathrm{x}_{3}^{\prime}
\end{array}
$$

hold for all $\mathrm{x}_{1}, \mathrm{x}_{1}^{\prime}, \mathrm{x}_{2}, \mathrm{x}_{2}^{\prime}, \mathrm{x}_{3}, \mathrm{x}_{3}^{\prime} \in \mathrm{N}$.

Lemma 3.4 : Let $\mathrm{N}$ be a near-ring and $\mathrm{d}$ be a two sided reverse $\alpha$-3-derivation of $\mathrm{N}$, then

(i) $\left(\alpha\left(\mathrm{x}_{1}\right) \mathrm{d}\left(\mathrm{x}_{1}^{\prime}, \mathrm{x}_{2}, \mathrm{x}_{3}\right)+\mathrm{d}\left(\mathrm{x}_{1}, \mathrm{x}_{2}, \mathrm{x}_{3}\right) \mathrm{x}_{1}^{\prime}\right) \mathrm{y}=\alpha\left(\mathrm{x}_{1}\right) \mathrm{d}\left(\mathrm{x}_{1}^{\prime}, \mathrm{x}_{2}, \mathrm{x}_{3}\right) \mathrm{y}+\mathrm{d}\left(\mathrm{x}_{1}, \mathrm{x}_{2}, \mathrm{x}_{3}\right) \mathrm{x}_{1}^{\prime} \mathrm{y}$ $\left(\alpha\left(\mathrm{x}_{2}\right) \mathrm{d}\left(\mathrm{x}_{1}, \mathrm{x}_{2}^{\prime}, \mathrm{x}_{3}\right)+\mathrm{d}\left(\mathrm{x}_{1}, \mathrm{x}_{2}, \mathrm{x}_{3}\right) \mathrm{x}_{2}^{\prime}\right) \mathrm{y}=\alpha\left(\mathrm{x}_{2}\right) \mathrm{d}\left(\mathrm{x}_{1}, \mathrm{x}_{2}^{\prime}, \mathrm{x}_{3}\right) \mathrm{y}+\mathrm{d}\left(\mathrm{x}_{1}, \mathrm{x}_{2}, \mathrm{x}_{3}\right) \mathrm{x}_{2}^{\prime} \mathrm{y}$ $\left(\alpha\left(\mathrm{x}_{3}\right) \mathrm{d}\left(\mathrm{x}_{1}, \mathrm{x}_{2}, \mathrm{x}_{3}^{\prime}\right)+\mathrm{d}\left(\mathrm{x}_{1}, \mathrm{x}_{2}, \mathrm{x}_{3}\right) \mathrm{x}_{3}^{\prime}\right) \mathrm{y}=\alpha\left(\mathrm{x}_{3}\right) \mathrm{d}\left(\mathrm{x}_{1}, \mathrm{x}_{2}, \mathrm{x}_{3}^{\prime}\right) \mathrm{y}+\mathrm{d}\left(\mathrm{x}_{1}, \mathrm{x}_{2}, \mathrm{x}_{3}\right) \mathrm{x}_{3}^{\prime} \mathrm{y}$ for all $\mathrm{x}_{1}, \mathrm{x}_{1}^{\prime}, \mathrm{x}_{2}, \mathrm{x}_{2}^{\prime}, \mathrm{x}_{3}, \mathrm{x}_{3}^{\prime}, \mathrm{y} \in \mathrm{N}$.

(ii) $\left(\mathrm{x}_{1} \mathrm{~d}\left(\mathrm{x}_{1}^{\prime}, \mathrm{x}_{2}, \mathrm{x}_{3}\right)+\mathrm{d}\left(\mathrm{x}_{1}, \mathrm{x}_{2}, \mathrm{x}_{3}\right) \alpha\left(\mathrm{x}_{1}^{\prime}\right)\right) \mathrm{y}=\mathrm{x}_{1} \mathrm{~d}\left(\mathrm{x}_{1}^{\prime}, \mathrm{x}_{2}, \mathrm{x}_{3}\right) \mathrm{y}+\mathrm{d}\left(\mathrm{x}_{1}, \mathrm{x}_{2}, \mathrm{x}_{3}\right) \alpha\left(\mathrm{x}_{1}^{\prime}\right) \mathrm{y}$ $\left(\mathrm{x}_{2} \mathrm{~d}\left(\mathrm{x}_{1}, \mathrm{x}_{2}^{\prime}, \mathrm{x}_{3}\right)+\mathrm{d}\left(\mathrm{x}_{1}, \mathrm{x}_{2}, \mathrm{x}_{3}\right) \alpha\left(\mathrm{x}_{2}^{\prime}\right)\right) \mathrm{y}=\mathrm{x}_{2} \mathrm{~d}\left(\mathrm{x}_{1}, \mathrm{x}_{2}^{\prime}, \mathrm{x}_{3}\right) \mathrm{y}+\mathrm{d}\left(\mathrm{x}_{1}, \mathrm{x}_{2}, \mathrm{x}_{3}\right) \alpha\left(\mathrm{x}_{2}^{\prime}\right) \mathrm{y}$ $\left(\mathrm{x}_{3} \mathrm{~d}\left(\mathrm{x}_{1}, \mathrm{x}_{2}, \mathrm{x}_{3}^{\prime}\right)+\mathrm{d}\left(\mathrm{x}_{1}, \mathrm{x}_{2}, \mathrm{x}_{3}\right) \alpha\left(\mathrm{x}_{3}^{\prime}\right)\right) \mathrm{y}=\mathrm{x}_{3} \mathrm{~d}\left(\mathrm{x}_{1}, \mathrm{x}_{2}, \mathrm{x}_{3}^{\prime}\right) \mathrm{y}+\mathrm{d}\left(\mathrm{x}_{1}, \mathrm{x}_{2}, \mathrm{x}_{3}\right) \alpha\left(\mathrm{x}_{3}^{\prime}\right) \mathrm{y}$ 
for all $\mathrm{x}_{1}, \mathrm{x}_{1}{ }^{\prime}, \mathrm{x}_{2}, \mathrm{x}_{2}{ }^{\prime}, \mathrm{x}_{3}, \mathrm{x}_{3}{ }^{\prime}, \mathrm{y} \in \mathrm{N}$.

Lemma 3.5 : Let $\mathrm{N}$ be a prime near-ring and $\mathrm{d}$ be a nonzero two sided reverse $\alpha$-3-derivation of $\mathrm{N}$ . Let $\mathrm{U}_{1}, \mathrm{U}_{2}, \mathrm{U}_{3}$ be a nonzero semigroup ideals of $\mathrm{N}$. If

$\mathrm{d}\left(\mathrm{U}_{1}, \mathrm{U}_{2}, \mathrm{U}_{3}\right)=\{0\}$, then $\mathrm{d}(\mathrm{N}, \mathrm{N}, \mathrm{N})=\{0\}$.

Now, we will prove the main results :

Theorem 3.6 : Let $\mathrm{N}$ be a prime near-ring and d be a nonzero two sided reverse $\alpha$-3-derivation of $\mathrm{N}$. Let $\mathrm{U}_{1}, \mathrm{U}_{2}, \mathrm{U}_{3}$ be a nonzero semigroup ideals of $\mathrm{N}$. If

$\mathrm{d}\left(\mathrm{U}_{1}, \mathrm{U}_{2}, \mathrm{U}_{3}\right) \subseteq \mathrm{Z}$, then $\mathrm{N}$ is a commutative ring .

Proof: We are given that

$\mathrm{d}\left(\mathrm{u}_{1}, \mathrm{u}_{2}, \mathrm{u}_{3}\right) \in \mathrm{Z}$ for all $\mathrm{u}_{1} \in \mathrm{U}_{1}, \mathrm{u}_{2} \in \mathrm{U}_{2}, \mathrm{u}_{3} \in \mathrm{U}_{3}$.

Therefore

$\mathrm{td}\left(\mathrm{u}_{1} / \mathrm{u}_{1}, \mathrm{u}_{2}, \mathrm{u}_{3}\right)=\mathrm{d}\left(\mathrm{u}_{1} / \mathrm{u}_{1}, \mathrm{u}_{2}, \mathrm{u}_{3}\right) \mathrm{t}$ for all $\mathrm{u}_{1}, \mathrm{u}_{1} / \in \mathrm{U}_{1}, \mathrm{u}_{2} \in \mathrm{U}_{2}, \mathrm{u}_{3} \in \mathrm{U}_{3}, \mathrm{t} \in \mathrm{N}$.

By Lemma 3.4 (ii) and defining property of $d$ we get

$\mathrm{t} \mathrm{u}_{1} \mathrm{~d}\left(\mathrm{u}_{1}{ }^{\prime}, \mathrm{u}_{2}, \mathrm{u}_{3}\right)+\mathrm{td}\left(\mathrm{u}_{1}, \mathrm{u}_{2}, \mathrm{u}_{3}\right) \alpha\left(\mathrm{u}_{1^{\prime}}\right)$

$=\mathrm{u}_{1} \mathrm{~d}\left(\mathrm{u}_{1}^{\prime}, \mathrm{u}_{2}, \mathrm{u}_{3}\right) \mathrm{t}+\mathrm{d}\left(\mathrm{u}_{1}, \mathrm{u}_{2}, \mathrm{u}_{3}\right) \alpha\left(\mathrm{u}_{1}^{\prime}\right) \mathrm{t}$

for all $\mathrm{u}_{1}, \mathrm{u}_{1}^{\prime} \in \mathrm{U}_{1}, \mathrm{u}_{2} \in \mathrm{U}_{2}, \mathrm{u}_{3} \in \mathrm{U}_{3}, \mathrm{t} \in \mathrm{N}$. Using (3.1) again , we obtain

$\mathrm{d}\left(\mathrm{u}_{1}{ }^{\prime}, \mathrm{u}_{2}, \mathrm{u}_{3}\right) \mathrm{t} \mathrm{u}_{1}+\mathrm{d}\left(\mathrm{u}_{1}, \mathrm{u}_{2}, \mathrm{u}_{3}\right) t \alpha\left(\mathrm{u}_{1}^{\prime}\right)=\mathrm{d}\left(\mathrm{u}_{1}{ }^{\prime}, \mathrm{u}_{2}, \mathrm{u}_{3}\right) \mathrm{u}_{1} \mathrm{t}+\mathrm{d}\left(\mathrm{u}_{1}, \mathrm{u}_{2}, \mathrm{u}_{3}\right) \alpha\left(\mathrm{u}_{1}{ }^{\prime}\right) \mathrm{t}$

for all $\mathrm{u}_{1}, \mathrm{u}_{1}^{\prime} \in \mathrm{U}_{1}, \mathrm{u}_{2} \in \mathrm{U}_{2}, \mathrm{u}_{3} \in \mathrm{U}_{3}, \mathrm{t} \in \mathrm{N}$.

Replacing t by $\alpha\left(\mathrm{u}_{1}^{\prime}\right)$ in (3.2), we get

$\mathrm{d}\left(\mathrm{u}_{1^{\prime}}, \mathrm{u}_{2}, \mathrm{u}_{3}\right) \alpha\left(\mathrm{u}_{1^{\prime}}\right) \mathrm{u}_{1}=\mathrm{d}\left(\mathrm{u}_{1}^{\prime}, \mathrm{u}_{2}, \mathrm{u}_{3}\right) \mathrm{u}_{1} \alpha\left(\mathrm{u}_{1}^{\prime}\right)$ for all $\mathrm{u}_{1}, \mathrm{u}_{1}^{\prime} \in \mathrm{U}_{1}, \mathrm{u}_{2} \in \mathrm{U}_{2}, \mathrm{u}_{3} \in \mathrm{U}_{3}$.

i.e.; $\mathrm{d}\left(\mathrm{u}_{1^{\prime}}{ }^{\prime}, \mathrm{u}_{2}, \mathrm{u}_{3}\right) \mathrm{N}\left[\alpha\left(\mathrm{u}_{1^{\prime}}\right), \mathrm{u}_{1}\right]=\{0\}$ for all $\mathrm{u}_{1}, \mathrm{u}_{1}^{\prime} \in \mathrm{U}_{1}, \mathrm{u}_{2} \in \mathrm{U}_{2}, \mathrm{u}_{3} \in \mathrm{U}_{3}$.

Primeness of $N$ yields that for each $u_{1} / \in U_{1}$, we get either $d\left(u_{1}{ }^{\prime}, u_{2}, u_{3}\right)=0$ for all

$\mathrm{u}_{2} \in \mathrm{U}_{2}, \mathrm{u}_{3} \in \mathrm{U}_{3}$ or $\left[\alpha\left(\mathrm{u}_{1^{\prime}}\right), \mathrm{u}_{1}\right]=0$ for all $\mathrm{u}_{1} \in \mathrm{U}_{1}$.

If $\mathrm{d}\left(\mathrm{u}_{1}{ }^{\prime}, \mathrm{u}_{2}, \mathrm{u}_{3}\right)=0$ for all $\mathrm{u}_{1}{ }^{\prime} \in \mathrm{U}_{1}, \mathrm{u}_{2} \in \mathrm{U}_{2}, \mathrm{u}_{3} \in \mathrm{U}_{3}$, then by Lemma 3.5 we conclude that $\mathrm{d}(\mathrm{N}, \mathrm{N}, \mathrm{N})=\{0\}$, leading to a contradiction as $\mathrm{d}$ is a nonzero two sided reverse $\alpha$-3-derivation of $\mathrm{N}$. Therefore there exist $\mathrm{x}_{1} \in \mathrm{U}_{1}, \mathrm{x}_{2} \in \mathrm{U}_{2}, \mathrm{x}_{3} \in \mathrm{U}_{3}$ all being nonzero such that $\mathrm{d}\left(\mathrm{x}_{1}, \mathrm{x}_{2}, \mathrm{x}_{3}\right) \neq 0$ and $\alpha\left(\mathrm{x}_{1}\right) \mathrm{u}=\mathrm{u} \alpha\left(\mathrm{x}_{1}\right)$ for all $\mathrm{u} \in \mathrm{U}_{1}$, replacing $\mathrm{u}$ by ut where $\mathrm{t} \in \mathrm{N}$, we get

$\mathrm{U}_{1}\left[\alpha\left(\mathrm{x}_{1}\right), \mathrm{t}\right]=\{0\}$, for all $\mathrm{t} \in \mathrm{N}$. By Lemma 2.13 we get $\alpha\left(\mathrm{x}_{1}\right) \in \mathrm{Z}$.

Taking $\mathrm{x}_{1}$ instead of $\mathrm{u}_{1}{ }^{\prime}, \mathrm{x}_{2}$ instead of $\mathrm{u}_{2}, \mathrm{x}_{3}$ instead of $\mathrm{u}_{3}$ in (3.2), we obtain

$\mathrm{d}\left(\mathrm{x}_{1}, \mathrm{x}_{2}, \mathrm{x}_{3}\right) \mathrm{tu}_{1}=\mathrm{d}\left(\mathrm{x}_{1}, \mathrm{x}_{2}, \mathrm{x}_{3}\right) \mathrm{u}_{1} \mathrm{t}$ for all $\mathrm{u}_{1} \in \mathrm{U}_{1}, \mathrm{t} \in \mathrm{N}$.

i.e.; $d\left(\mathrm{x}_{1}, \mathrm{x}_{2}, \mathrm{x}_{3}\right)\left[\mathrm{t}, \mathrm{u}_{1}\right]=0$, accordingly

$\mathrm{d}\left(\mathrm{x}_{1}, \mathrm{x}_{2}, \mathrm{x}_{3}\right) \mathrm{N}\left[\mathrm{t}, \mathrm{u}_{1}\right]=\{0\}$ for all $\mathrm{u}_{1} \in \mathrm{U}_{1}, \mathrm{t} \in \mathrm{N}$.

Primeness of $\mathrm{N}$ and $\mathrm{d}\left(\mathrm{x}_{1}, \mathrm{x}_{2}, \mathrm{x}_{3}\right) \neq 0$ yield that $\mathrm{U}_{1} \subseteq \mathrm{Z}$, by Lemma 2.15 we conclude that $\mathrm{N}$ is a commutative ring .

Theorem 3.7 : Let $\mathrm{N}$ be a prime near-ring admitting a generalized two sided reverse $\alpha$-3derivation fassociated with a nonzero two sided reverse $\alpha$-3-derivation $d$. Let $\mathrm{U}_{1}, \mathrm{U}_{2}, \mathrm{U}_{3}$ be a nonzero semigroup ideals of $\mathrm{N}$. If $\mathrm{f}\left(\left[\mathrm{u}_{1}, \mathrm{u}_{1}^{\prime}\right], \mathrm{u}_{2}, \mathrm{u}_{3}\right)=0$, for all $\mathrm{u}_{1}, \mathrm{u}_{1}^{\prime} \in \mathrm{U}_{1}, \mathrm{u}_{2} \in \mathrm{U}_{2}, \mathrm{u}_{3} \in \mathrm{U}_{3}$, then $\mathrm{N}$ is a commutative ring .

Proof: By our hypothesis we have

$\mathrm{f}\left(\left[\mathrm{u}_{1}, \mathrm{u}_{1}^{\prime}\right], \mathrm{u}_{2}, \mathrm{u}_{3}\right)=0$, for all $\mathrm{u}_{1}, \mathrm{u}_{1}^{\prime} \in \mathrm{U}_{1}, \mathrm{u}_{2} \in \mathrm{U}_{2}, \mathrm{u}_{3} \in \mathrm{U}_{3}$.

Replacing $\mathrm{u}_{1} /$ by $\mathrm{u}_{1} / \mathrm{u}_{1}$ in preceding equation and using it again we get

$\mathrm{d}\left(\mathrm{u}_{1}, \mathrm{u}_{2}, \mathrm{u}_{3}\right)\left[\mathrm{u}_{1}, \mathrm{u}_{1}^{\prime}\right]=0$, for all $\mathrm{u}_{1}, \mathrm{u}_{1}^{\prime} \in \mathrm{U}_{1}, \mathrm{u}_{2} \in \mathrm{U}_{2}, \mathrm{u}_{3} \in \mathrm{U}_{3}$.

i.e.; $\mathrm{d}\left(\mathrm{u}_{1}, \mathrm{u}_{2}, \mathrm{u}_{3}\right) \mathrm{u}_{1} \mathrm{u}_{1}^{\prime}=\mathrm{d}\left(\mathrm{u}_{1}, \mathrm{u}_{2}, \mathrm{u}_{3}\right) \mathrm{u}_{1}^{\prime} \mathrm{u}_{1}$,

for all $\mathrm{u}_{1}, \mathrm{u}_{1}^{\prime} \in \mathrm{U}_{1}, \mathrm{u}_{2} \in \mathrm{U}_{2}, \mathrm{u}_{3} \in \mathrm{U}_{3}$ 
Replacing $\mathrm{u}_{1} /$ by $\mathrm{u}_{1} / \mathrm{r}$, where $\mathrm{r} \in \mathrm{N}$, in (3.3) and using it again we get $\mathrm{d}\left(\mathrm{u}_{1}, \mathrm{u}_{2}, \mathrm{u}_{3}\right) \mathrm{u}_{1}^{\prime}\left[\mathrm{u}_{1}, \mathrm{r}\right]=0$, for all $\mathrm{u}_{1}, \mathrm{u}_{1}{ }^{\prime} \in \mathrm{U}_{1}, \mathrm{u}_{2} \in \mathrm{U}_{2}, \mathrm{u}_{3} \in \mathrm{U}_{3}, \mathrm{r} \in \mathrm{N}$.

Therefore $\mathrm{d}\left(\mathrm{u}_{1}, \mathrm{u}_{2}, \mathrm{u}_{3}\right) \mathrm{U}_{1}\left[\mathrm{u}_{1}, \mathrm{r}\right]=\{0\}$, for all $\mathrm{u}_{1} \in \mathrm{U}_{1}, \mathrm{u}_{2} \in \mathrm{U}_{2}, \mathrm{u}_{3} \in \mathrm{U}_{3}, r \in N$.

By Lemma 2.14, we conclude that for each $\mathrm{u}_{1} \in \mathrm{U}_{1}$ either $\mathrm{u}_{1} \in \mathrm{Z}$ or $\mathrm{d}\left(\mathrm{u}_{1}, \mathrm{u}_{2}, \mathrm{u}_{3}\right)=0$

for all $\mathrm{u}_{2} \in \mathrm{U}_{2}, \mathrm{u}_{3} \in \mathrm{U}_{3}$.

Let $\mathrm{x}_{1} \in \mathrm{U}_{1} \cap \mathrm{Z}$, by Lemma 3.3 and defining property of $\mathrm{d}$, we have for all $\mathrm{y} \in \mathrm{N}$.

$\mathrm{d}\left(\mathrm{yx}_{1}, \mathrm{u}_{2}, \mathrm{u}_{3}\right)=\mathrm{x}_{1} \mathrm{~d}\left(\mathrm{y}, \mathrm{u}_{2}, \mathrm{u}_{3}\right)+\mathrm{d}\left(\mathrm{x}_{1}, \mathrm{u}_{2}, \mathrm{u}_{3}\right) \alpha(\mathrm{y})$

$=\mathrm{d}\left(\mathrm{x}_{1} \mathrm{y}, \mathrm{u}_{2}, \mathrm{u}_{3}\right)$

$=\mathrm{d}\left(\mathrm{y}, \mathrm{u}_{2}, \mathrm{u}_{3}\right) \mathrm{x}_{1}+\alpha(\mathrm{y}) \mathrm{d}\left(\mathrm{x}_{1}, \mathrm{u}_{2}, \mathrm{u}_{3}\right)$

for all $\mathrm{u}_{2} \in \mathrm{U}_{2}, \mathrm{u}_{3} \in \mathrm{U}_{3}, \mathrm{y} \in \mathrm{N}$. Which implies that

$\mathrm{d}\left(\mathrm{x}_{1}, \mathrm{u}_{2}, \mathrm{u}_{3}\right) \alpha(\mathrm{y})=\alpha(\mathrm{y}) \mathrm{d}\left(\mathrm{x}_{1}, \mathrm{u}_{2}, \mathrm{u}_{3}\right)$

for all $\mathrm{u}_{2} \in \mathrm{U}_{2}, \mathrm{u}_{3} \in \mathrm{U}_{3}, \mathrm{y} \in \mathrm{N}$.

In view of equation (3.4) we get

$\mathrm{d}\left(\mathrm{u}_{1}, \mathrm{u}_{2}, \mathrm{u}_{3}\right) \alpha(\mathrm{y})=\alpha(\mathrm{y}) \mathrm{d}\left(\mathrm{u}_{1}, \mathrm{u}_{2}, \mathrm{u}_{3}\right)$

for all $\mathrm{u}_{1} \in \mathrm{U}_{1}, \mathrm{u}_{2} \in \mathrm{U}_{2}, \mathrm{u}_{3} \in \mathrm{U}_{3}, \mathrm{y} \in \mathrm{N}$.

On the other hand,

$\mathrm{d}\left(\mathrm{tx}_{1}, \mathrm{u}_{2}, \mathrm{u}_{3}\right)=\mathrm{d}\left(\mathrm{x}_{1}, \mathrm{u}_{2}, \mathrm{u}_{3}\right) \mathrm{t}+\alpha\left(\mathrm{x}_{1}\right) \mathrm{d}\left(\mathrm{t}, \mathrm{u}_{2}, \mathrm{u}_{3}\right)=\mathrm{d}\left(\mathrm{x}_{1} \mathrm{t}, \mathrm{u}_{2}, \mathrm{u}_{3}\right)$

$$
=\operatorname{td}\left(\mathrm{x}_{1}, \mathrm{u}_{2}, \mathrm{u}_{3}\right)+\mathrm{d}\left(\mathrm{t}, \mathrm{u}_{2}, \mathrm{u}_{3}\right) \alpha\left(\mathrm{x}_{1}\right)
$$

for all $\mathrm{u}_{2} \in \mathrm{U}_{2}, \mathrm{u}_{3} \in \mathrm{U}_{3}, \mathrm{t} \in \mathrm{N}$.

It follows that for all $\mathrm{u}_{2} \in \mathrm{U}_{2}, \mathrm{u}_{3} \in \mathrm{U}_{3}, \mathrm{t} \in \mathrm{N}$, we get

$\mathrm{d}\left(\mathrm{x}_{1}, \mathrm{u}_{2}, \mathrm{u}_{3}\right) \mathrm{t}+\alpha\left(\mathrm{x}_{1}\right) \mathrm{d}\left(\mathrm{t}, \mathrm{u}_{2}, \mathrm{u}_{3}\right)=\mathrm{td}\left(\mathrm{x}_{1}, \mathrm{u}_{2}, \mathrm{u}_{3}\right)+\mathrm{d}\left(\mathrm{t}, \mathrm{u}_{2}, \mathrm{u}_{3}\right) \alpha\left(\mathrm{x}_{1}\right)$

In particular, taking $t \in \mathrm{U}_{1}$ in (3.6) and using (3.5), we get

$\mathrm{d}\left(\mathrm{x}_{1}, \mathrm{u}_{2}, \mathrm{u}_{3}\right) \mathrm{t}=\mathrm{td}\left(\mathrm{x}_{1}, \mathrm{u}_{2}, \mathrm{u}_{3}\right)$ for all $\mathrm{t} \in \mathrm{U}_{1}, \mathrm{u}_{2} \in \mathrm{U}_{2}, \mathrm{u}_{3} \in \mathrm{U}_{3}$.

Replacing $t$ by ty, where $y \in N$, in the preceding equation and using it again to get

$\operatorname{tyd}\left(\mathrm{x}_{1}, \mathrm{u}_{2}, \mathrm{u}_{3}\right)=\mathrm{d}\left(\mathrm{x}_{1}, \mathrm{u}_{2}, \mathrm{u}_{3}\right) \mathrm{ty}=\operatorname{td}\left(\mathrm{x}_{1}, \mathrm{u}_{2}, \mathrm{u}_{3}\right) \mathrm{y}$

for all $t \in U_{1}, u_{2} \in U_{2}, u_{3} \in U_{3}, y \in N$, that is

$\mathrm{t}\left[\mathrm{d}\left(\mathrm{x}_{1}, \mathrm{u}_{2}, \mathrm{u}_{3}\right), \mathrm{y}\right]=0$ for all $\mathrm{t} \in \mathrm{U}_{1}, \mathrm{u}_{2} \in \mathrm{U}_{2}, \mathrm{u}_{3} \in \mathrm{U}_{3}, \mathrm{y} \in \mathrm{N}$.

Therefore

$\mathrm{U}_{1}\left[\mathrm{~d}\left(\mathrm{x}_{1}, \mathrm{u}_{2}, \mathrm{u}_{3}\right), \mathrm{y}\right]=\{0\}$, by Lemma 2.13 we get $\mathrm{d}\left(\mathrm{x}_{1}, \mathrm{u}_{2}, \mathrm{u}_{3}\right) \in \mathrm{Z}$. According to (3.4) we conclude that $d\left(u_{1}, u_{2}, u_{3}\right) \in Z$ for all $u_{1} \in U_{1}, u_{2} \in U_{2}, u_{3} \in U_{3}$, and hence $N$ is a commutative ring by application of Theorem 3.6 .

Corollary 3.8 : Let $\mathrm{N}$ be a prime near-ring admitting a nonzero two sided reverse $\alpha$-3-derivation $\mathrm{d}$ . Let $\mathrm{U}_{1}, \mathrm{U}_{2}, \mathrm{U}_{3}$ be a nonzero semigroup ideals of $\mathrm{N}$. If $\mathrm{d}\left(\left[\mathrm{u}_{1}, \mathrm{u}_{1}^{\prime}\right], \mathrm{u}_{2}, \mathrm{u}_{3}\right)=0$, for all $\mathrm{u}_{1}, \mathrm{u}_{1}^{\prime} \in$ $\mathrm{U}_{1}, \mathrm{u}_{2} \in \mathrm{U}_{2}, \mathrm{u}_{3} \in \mathrm{U}_{3}$, then $\mathrm{N}$ is a commutative ring .

Theorem 3.9: Let $\mathrm{N}$ be a prime near-ring admitting a nonzero generalized two sided reverse $\alpha$-3derivation fassociated with a nonzero two sided reverse $\alpha$-3-derivation d of N.Let $\mathrm{U}_{1}, \mathrm{U}_{2}, \mathrm{U}_{3}$ be a nonzero semigroup ideals of N.If $\mathrm{f}\left(\left[\mathrm{u}_{1}, \mathrm{u}_{1}^{\prime}\right], \mathrm{u}_{2}, \mathrm{u}_{3}\right)= \pm\left[\mathrm{u}_{1}, \mathrm{u}_{1}^{\prime}\right]$, for all $\mathrm{u}_{1}, \mathrm{u}_{1}^{\prime} \in \mathrm{U}_{1}, \mathrm{u}_{2} \in$ $\mathrm{U}_{2}, \mathrm{u}_{3} \in \mathrm{U}_{3}$, then $\mathrm{N}$ is a commutative ring .

Proof: By our hypothesis, we have

$\mathrm{f}\left(\left[\mathrm{u}_{1}, \mathrm{u}_{1}^{\prime}\right], \mathrm{u}_{2}, \mathrm{u}_{3}\right)= \pm\left[\mathrm{u}_{1}, \mathrm{u}_{1}^{\prime}\right]$, for all $\mathrm{u}_{1}, \mathrm{u}_{1}^{\prime} \in \mathrm{U}_{1}, \mathrm{u}_{2} \in \mathrm{U}_{2}, \mathrm{u}_{3} \in \mathrm{U}_{3}$.

Replacing $\mathrm{u}_{1} /$ by $\mathrm{u}_{1} / \mathrm{u}_{1}$ in (3.7) and using it again we get

$\mathrm{d}\left(\mathrm{u}_{1}, \mathrm{u}_{2}, \mathrm{u}_{3}\right) \alpha\left(\left[\mathrm{u}_{1}, \mathrm{u}_{1}^{\prime}\right]\right)=0$, for all $\mathrm{u}_{1}, \mathrm{u}_{1}^{\prime} \in \mathrm{U}_{1}, \mathrm{u}_{2} \in \mathrm{U}_{2}, \mathrm{u}_{3} \in \mathrm{U}_{3}$.

i.e.; $\mathrm{d}\left(\mathrm{u}_{1}, \mathrm{u}_{2}, \mathrm{u}_{3}\right) \alpha\left(\mathrm{u}_{1}\right) \alpha\left(\mathrm{u}_{1}^{\prime}\right)=\mathrm{d}\left(\mathrm{u}_{1}, \mathrm{u}_{2}, \mathrm{u}_{3}\right) \alpha\left(\mathrm{u}_{1}^{\prime}\right) \alpha\left(\mathrm{u}_{1}\right)$, let $\alpha\left(\mathrm{U}_{1}\right)=\mathrm{V}_{1}$ since $\alpha$ is surjective , then $\mathrm{V}_{1}$ is a semigroup ideal of $\mathrm{N}$. Now let $\alpha\left(\mathrm{u}_{1}^{\prime}\right)=\mathrm{v}_{1}^{\prime}$, where $\mathrm{v}_{1}{ }^{\prime} \in \mathrm{V}_{1}$ so we have for all $\mathrm{u}_{1} \in$ $\mathrm{U}_{1}, \mathrm{u}_{2} \in \mathrm{U}_{2}, \mathrm{u}_{3} \in \mathrm{U}_{3}, \mathrm{v}_{1}^{\prime} \in \mathrm{V}_{1}$. 
$\mathrm{d}\left(\mathrm{u}_{1}, \mathrm{u}_{2}, \mathrm{u}_{3}\right) \alpha\left(\mathrm{u}_{1}\right) \mathrm{v}_{1}^{\prime}=\mathrm{d}\left(\mathrm{u}_{1}, \mathrm{u}_{2}, \mathrm{u}_{3}\right) \mathrm{v}_{1}^{\prime} \alpha\left(\mathrm{u}_{1}\right)$

Replacing $\mathrm{v}_{1} /$ by $\mathrm{v}_{1} / \mathrm{r}$, where $\mathrm{r} \in \mathrm{N}$, in (3.8) and using it again we get

$\mathrm{d}\left(\mathrm{u}_{1}, \mathrm{u}_{2}, \mathrm{u}_{3}\right) \mathrm{v}_{1}{ }^{\prime}\left[\alpha\left(\mathrm{u}_{1}\right), \mathrm{r}\right]=0$ for all $\mathrm{u}_{1} \in \mathrm{U}_{1}, \mathrm{u}_{2} \in \mathrm{U}_{2}, \mathrm{u}_{3} \in \mathrm{U}_{3}, \mathrm{v}_{1} / \in \mathrm{V}_{1}, \mathrm{r} \in \mathrm{N}$.

i.e.; $\mathrm{d}\left(\mathrm{u}_{1}, \mathrm{u}_{2}, \mathrm{u}_{3}\right) \mathrm{V}_{1}\left[\alpha\left(\mathrm{u}_{1}\right), \mathrm{r}\right]=\{0\}$ for all $\mathrm{u}_{1} \in \mathrm{U}_{1}, \mathrm{u}_{2} \in \mathrm{U}_{2}, \mathrm{u}_{3} \in \mathrm{U}_{3}, \mathrm{r} \in \mathrm{N}$.

By Lemma 2.14, we get for all $\mathrm{u}_{1} \in \mathrm{U}_{1}$, either $\alpha\left(\mathrm{u}_{1}\right) \in \mathrm{Z}$ or $\mathrm{d}\left(\mathrm{u}_{1}, \mathrm{u}_{2}, \mathrm{u}_{3}\right)=0$

for all $\mathrm{u}_{2} \in \mathrm{U}_{2}, \mathrm{u}_{3} \in \mathrm{U}_{3}$

Let $u \in U_{1}$ such that $d\left(u, u_{2}, u_{3}\right)=0$, for all $u_{2} \in U_{2}, u_{3} \in U_{3}$, then

$\mathrm{d}\left(\mathrm{uu}^{\prime}, \mathrm{u}_{2}, \mathrm{u}_{3}\right)=\mathrm{d}\left(\mathrm{u}^{\prime}, \mathrm{u}_{2}, \mathrm{u}_{3}\right) \mathrm{u}+\alpha\left(\mathrm{u}^{\prime}\right) \mathrm{d}\left(\mathrm{u}, \mathrm{u}_{2}, \mathrm{u}_{3}\right)=\mathrm{d}\left(\mathrm{u}^{\prime}, \mathrm{u}_{2}, \mathrm{u}_{3}\right) \mathrm{u}$ and

$\mathrm{d}\left(\mathrm{uu}^{\prime}, \mathrm{u}_{2}, \mathrm{u}_{3}\right)=\mathrm{d}\left(\mathrm{u}^{\prime}, \mathrm{u}_{2}, \mathrm{u}_{3}\right) \alpha(\mathrm{u})+\mathrm{u}^{\prime} \mathrm{d}\left(\mathrm{u}, \mathrm{u}_{2}, \mathrm{u}_{3}\right)=\mathrm{d}\left(\mathrm{u}^{\prime}, \mathrm{u}_{2}, \mathrm{u}_{3}\right) \alpha(\mathrm{u})$

for all $\mathrm{u}^{\prime} \in \mathrm{U}_{1}, \mathrm{u}_{2} \in \mathrm{U}_{2}, \mathrm{u}_{3} \in \mathrm{U}_{3}$.

Combining both expressions of $\mathrm{d}\left(\mathrm{uu}^{\prime}, \mathrm{u}_{2}, \mathrm{u}_{3}\right)$, we obtain

$\mathrm{d}\left(\mathrm{u}^{\prime}, \mathrm{u}_{2}, \mathrm{u}_{3}\right)(\alpha(\mathrm{u})-\mathrm{u})=0$ for all $\mathrm{u}^{\prime} \in \mathrm{U}_{1}, \mathrm{u}_{2} \in \mathrm{U}_{2}, \mathrm{u}_{3} \in \mathrm{U}_{3}$.

Replacing $\mathrm{u}^{\prime}$ by $\mathrm{wu}^{\prime}$, where $\mathrm{w} \in \mathrm{U}_{1}$, in (3.10) and using it again to get

$\mathrm{d}\left(\mathrm{u}^{\prime}, \mathrm{u}_{2}, \mathrm{u}_{3}\right) w(\alpha(\mathrm{u})-\mathrm{u})=0$ for all $\mathrm{u}^{\prime}, \mathrm{w} \in \mathrm{U}_{1}, \mathrm{u}_{2} \in \mathrm{U}_{2}, \mathrm{u}_{3} \in \mathrm{U}_{3}$.

i.e.; $d\left(u^{\prime}, u_{2}, u_{3}\right) U_{1}(\alpha(u)-u)=\{0\}$ for all $u^{\prime} \in U_{1}, u_{2} \in U_{2}, u_{3} \in U_{3}$.

By Lemma 2.14 we conclude that either $\mathrm{d}\left(\mathrm{u}^{\prime}, \mathrm{u}_{2}, \mathrm{u}_{3}\right)=0$ for all $\mathrm{u}^{\prime} \in \mathrm{U}_{1}, \mathrm{u}_{2} \in \mathrm{U}_{2}, \mathrm{u}_{3} \in \mathrm{U}_{3}$ or $\alpha(\mathrm{u})=\mathrm{u}$.

If $\mathrm{d}\left(\mathrm{u}^{\prime}, \mathrm{u}_{2}, \mathrm{u}_{3}\right)=0$ for all $\mathrm{u}^{\prime} \in \mathrm{U}_{1}, \mathrm{u}_{2} \in \mathrm{U}_{2}, \mathrm{u}_{3} \in \mathrm{U}_{3}$, then by Lemma 3.5 we conclude $\mathrm{d}=0$, which contradicts our original assumption that $\mathrm{d} \neq 0$.

Hence, we conclude that $\alpha(\mathrm{u})=\mathrm{u}$. According to (3.9) we arrive at a conclusion .

For each $\mathrm{u}_{1} \in \mathrm{U}_{1}$, either $\alpha\left(\mathrm{u}_{1}\right) \in \mathrm{Z}$ or $\mathrm{d}\left(\alpha\left(\mathrm{u}_{1}\right), \mathrm{u}_{2}, \mathrm{u}_{3}\right)=0$ for all $\mathrm{u}_{2} \in \mathrm{U}_{2}, \mathrm{u}_{3} \in \mathrm{U}_{3}$

It follows for all $\mathrm{v}_{1} \in \mathrm{V}_{1}$, we get either $\mathrm{v}_{1} \in \mathrm{Z}$ or $\mathrm{d}\left(\mathrm{v}_{1}, \mathrm{u}_{2}, \mathrm{u}_{3}\right)=0$ for all $\mathrm{u}_{2} \in \mathrm{U}_{2}, \mathrm{u}_{3} \in \mathrm{U}_{3}$. Which is identical with the equation (3.4) in Theorem 3.7. Now arguing in the same way in corollary 3.8 we conclude that $\mathrm{d}\left(\mathrm{v}_{1}, \mathrm{u}_{2}, \mathrm{u}_{3}\right) \in \mathrm{Z}$ for all $\mathrm{v}_{1} \in \mathrm{V}_{1}, \mathrm{u}_{2} \in \mathrm{U}_{2}, \mathrm{u}_{3} \in \mathrm{U}_{3}$, and hence $\mathrm{N}$ is a commutative ring by application of Theorem 3.6

\section{Conclusion}

In present paper we introduce the notions of two sided reverse $\alpha$-3-derivation and generalized two sided reverse $\alpha$-3-derivation in near-ring and we see that a near-ring can be make commutative with help of generalized two sided reverse $\alpha$-3-derivation and other conditions .

\section{References}

[1] I. N. Herstein , Topics in Ring Theory , University of Chicago Press , Chicago ,1969 .

[2] I. A. Saed , "On Semigroup Ideals and Left Generalized $(\theta, \theta)$-4-Derivations in Prime NearRings " , International Journal of Mathematics Trends and Technology , Vol.57, Issue 2 , May 2018

[3] I. A. Saed , "Right $(\theta, \theta)$-4-Derivations on Prime Near-Rings " , International Journal of Mathematics Trends and Technology, Vol.54 , No.3 ,February 2018.

[4] I. A. Saed , " On Semiprime Gamma Near-Rings with Perpendicular Generalized 3-Derivations " Journal of AL-Qadisiyah for computer science and mathematics , Vol.11, No.2 , 2019 .

[5] E.F.Adhab , " n-Additive Mapping in Prime and Semiprime Near-Rings " ,PHD . thesis , Baghdad University , Iraq , 2016. 
[6] N. Argac, "On near-rings with two sided $\alpha$-derivations " , Turk. J. Math. , 28, 195-204 , 2004.

[7] L. Oukhtite and A. Raji , " Generalized two sided $\alpha$-derivations in 3-prime near-rings ", J. Taibah University for science ,9,366-372,2015.

[8] M. Asraf. and M. A. Siddeeque, " On permuting n-derivations in near-rings " ,Commun. Kor. Math. Soc., 28(4), 697-707, 2013.

[9] M. Asraf. and M. A. Siddeeque , " On $(\sigma, \tau)$-n-derivations in near-rings " , Asian-European Journal of Mathematics , 6(4), 14 pages , 2013 .

[10] M. Asraf. and M. A. Siddeeque, " On generalized n-derivations in near-rings " , Palestine Journal of Mathematics ,3(1) ,468-480, 2014.

[11] M. Asraf. and M. A. Siddeeque and Parveen. N, " On semigroup ideals and n-derivations in near-rings ", Science Direct Journal of Taibah University for Science , 9 , 126-132, 2014.

[12] I. A. Saed , " Commutativity of Addition in Prime Near-Rings with Right $(\theta, \theta)$-3-Derivations" ,Journal of Advances in Mathematics , Vol.14 , Issuel 1 , 2018.

[13] I. A. Saed , " On Semigroup Ideals and $(\theta, \theta)$-3-Derivations in Near-Rings " , International Journal of Mathematics Trends and Technology, Vol.44 , No.1 , April 2017.

[14] I. A. Saed , " On Generalized $(\theta, \theta)$-3-Derivations in Prime Near-Rings " ,Journal of Progressive Research in Mathematics, Vol.12 , Issuel $1,2017$. 